\title{
Toward a BT.2020 green emitter through a combined multiple resonance effect and multi-lock strategy
}

\section{Junyuan Liu}

Zhejiang University

\section{Yunhui Zhu}

Zhejiang Hongwu Technology Co., Ltd.

\section{Taiju Tsuboi}

Zhejiang University

\section{Chao Deng}

Zhejiang Hongwu Technology Co., Ltd.

\section{Weiwei Lou}

Zhejiang Hongwu Technology Co., Ltd.

\section{Dan Wang}

Zhejiang University

\section{Tiangeng Liu}

Zhejiang University

Qisheng Zhang ( $\sim$ qishengzhang@zju.edu.cn )

Zhejiang University https://orcid.org/0000-0002-0899-6856

\section{Article}

Keywords:

Posted Date: March 1st, 2022

DOI: https://doi.org/10.21203/rs.3.rs-1357408/v1

License: (1) (1) This work is licensed under a Creative Commons Attribution 4.0 International License. Read Full License

Version of Record: A version of this preprint was published at Nature Communications on August 19th, 2022. See the published version at https://doi.org/10.1038/s41467-022-32607-3. 
Toward a BT.2020 green emitter through a combined multiple resonance effect and multi-lock strategy

Junyuan Liu, Yunhui Zhu*, Taiju Tsuboi, Chao Deng, Weiwei Lou, Dan Wang, Tiangeng Liu, Qisheng Zhang*

J. Liu, Dr. T. Tsuboi, Dr. D. Wang, T. Liu, Prof. Q. Zhang

MOE Key Laboratory of Macromolecular Synthesis and Functionalization, Department of Polymer Science and Engineering, Zhejiang University, Hangzhou 310027, P.R. China

Dr. Y. Zhu, Dr. C. Deng, W. Lou

Zhejiang Hongwu Technology Co., Ltd., Taizhou 317100, P.R. China

Prof. Q. Zhang

State Key Laboratory of Clean Energy Utilization, Zhejiang University, Hangzhou 310027, P.R.

China

\section{Contributions}

Q.Z. initiated and supervised the project. J.L. and H.Z. designed, synthesized and characterized the MR-TADF green emitters. J.L. performed the computational calculation, photophysical and electrochemical measurements of the MR-TADF emitters. J.L. and W.L. fabricated the OLEDs and measured the device performance. J.L. and Q.Z. contributed to the manuscript writing. T.T., C.D., D.W., T.L. and Q.Z. provided suggestions on experiments and writing manuscript. All authors discussed the 
progress of the research and reviewed the manuscript.

Corresponding authors

Correspondence to:

Prof. Q. Zhang (E-mail: qishengzhang@zju.edu.cn)

Dr. H. Zhu (E-mail: zhuyunhui@hwoled.com)

\section{Abstract}

Color-saturated green-emitting molecules with high Commission Internationale de L'Eclairage (CIE) y values have great potential applications for displays and imaging. In this study, we linked the outer phenyl groups in multiple-resonance (MR)-type blue-emitting B (boron)-N (nitrogen) molecules through bonding and spiro-carbon bridges, which resulted in planar and rigid green emitters with thermally activated delayed fluorescence. The MR effect and multiple interlocking strategy greatly suppressed the high-frequency vibrations in the molecules, which emit green light with a full-width at half-maximum of $14 \mathrm{~nm}$ and a CIE y value of 0.77 in cyclohexane. These were the purest green molecules with quantum efficiency and color purity that were comparable to the best quantum dots currently available. Doping these emitters into a traditional green-emitting phosphorescence organic light-emitting diode (OLED) endowed the device with a Broadcast Service Television 2020 color-gamut, 50\% improved external quantum efficiency, and an extremely high luminescence of $4.6 \times 10^{5} \mathrm{~cd} / \mathrm{m}^{2}$, making it the greenest and brightest OLED ever reported.

\section{Introduction}


To better reproduce the true colors in the world, the color-gamut standard in display technology has gradually advanced from standard RGB (sRGB) to the National Television Standards Committee (NTSC), and now to the Broadcast Service Television 2020 (BT.2020) standard, with the largest color-gamut triangle coverage for Commission Internationale de L'Eclairage (CIE) 1931 color space thus far. To achieve the BT.2020 standard for each of the three primary colors, the emission maximum should be in a specific wavelength region, and the full width at half-maximum (FWHM) should be sufficiently narrow. Among the different types of luminescent materials, quantum dots (QDs) possess extremely high color purity by controlling their nanoscale size. ${ }^{1-3}$ However, the high cost and difficulty of their process control and storage hinder the large scale applications of QDs. ${ }^{4}$ The design and synthesis of organic fluorescent molecules with high color purity are both attractive and challenging. The use of narrow-band emitters in organic light-emitting diode (OLED) displays can reduce light loss caused by the color filter and/or optical microcavity, ${ }^{5,6}$ thereby improving the efficiency and stability of the displays.

Generally, the emission spectra of fluorescent molecules are broad due to structural deformation in the excited state and the molecular vibrations, ${ }^{7-12}$ which are enhanced by thermal conductivity through conjugated $\pi$-bonds. ${ }^{13,14}$ Recently, Hatakeyama et al. reported on a new kind of thermally activated delayed fluorescence (TADF) emitters based on multiple-resonance (MR). ${ }^{15-17}$ The separation of the highest occupied (HOMO) and lowest unoccupied (LUMO) molecular orbitals at the atomic level resulted in small singlet-triplet exchange energy, ${ }^{18}$ as well as suppressed thermal conductivity in the excited state. The spectral FWHM value of the devices containing a blue MR-TADF emitter, $v$-DABNA, 
reached $18 \mathrm{~nm}$, which was narrower than state-of-the-art micro-light-emitting diodes and QDs light-emitting diodes, and was quite close to the blue color requirements for the BT.2020 standard. ${ }^{18}$ Enhancing the electron-donating and -withdrawing capabilities of the fragments in the MR-TADF molecules can result in a bathochromic shift in the emission spectra. In 2019, researchers obtained a series of green MR-TADF emitters by introducing an electron-deficient group with fluoro-substituents into sky-blue emitting DtBuCzB (Scheme 1). ${ }^{19,20}$ In 2020, Duan et al. obtained a green MR-TADF emitter, AZA-BN, by introducing an electron-deficient aza-aromatics group to expand the molecular conjugation. ${ }^{21}$ The device containing AZA-BN achieved a CIE y value of 0.69 , which is currently the best performing bottom-emitting green organic light-emitting diode (OLED) to date. However, the above molecular design strategies enhanced the electronic structural changes as well as solid-state solvation in the excited state, which broadened the emission band along with the emission red-shift. ${ }^{19-22}$

In this work, we reported on two green MR-TADF molecules, tCzphB-Fl and tCzphB-Ph (Fig. 1), with emissions that can meet the BT.2020 color-gamut standard for photoluminescence (PL) and electroluminescence (EL). These two molecules were evolved from the blue MR-TADF emitter $t$-DABNA and the sky-blue emitter DtBuCzB. ${ }^{20,23} t$-DABNA contains two isolated phenyl groups that are perpendicular to the $\mathrm{BN}$-core plane. Wang et al. enlarged the $\pi$-conjugation length of $t$-DABNA by directly linking the phenyl groups and BN core through $\mathrm{C}-\mathrm{C}$ bonds and obtained sky-blue emitting DtBuCzB. ${ }^{20}$ However, the two outer phenyl-rings were twisted slightly out of the core plane in $\mathrm{DtBuCzB}$, due to steric repulsion between the adjacent H-atoms. In this work, two more planar green-light emitting molecules were 
obtained by locking the outer phenyl-rings with the central phenyl-ring in DtBuCzB using spiro-carbon formation, which differed from the previous design strategy through the introduction of electron-withdrawing groups. This new strategy suppressed the excited-state distortion and the vibration modes caused by the strong intramolecular charge transfer effect. ${ }^{7}$ The spectral FWHM values of these two molecules in cyclohexane solution were as narrow as $14 \mathrm{~nm}$. The OLEDs containing the new emitters achieved a purely green emission with an FWHM value of $24 \mathrm{~nm}$ and CIE coordinates of $(0.21,0.75)$, and this was the purest green emission reported for bottom-emitting OLEDs. Due to the TADF decay pathway for triplet excitons, the devices achieved a very high external quantum efficiency (EQE) of $31 \%$, which was $50 \%$ higher than the control devices based on classic green phosphorescent materials.

\section{Results}

Characterization of MR-TADF green emitters. $\mathrm{tCzphB}-\mathrm{Ph}$ and $\mathrm{tCzphB}-\mathrm{Fl}$ were prepared with good yields using two-step cascade reactions from 1,5-dibromo-2,4-difluorobenzene and 9,9'-(4,6-dibromo-1,3-phenylene) bis-9H-carbazole, as shown in Supplementary Fig. 10. These two compounds showed very small Stokes shifts of $15 \mathrm{~nm}$ in toluene solution $\left(1 \times 10^{-5}\right.$ M) at room temperature (RT) (Fig. 2a), implying very small geometric distortion in the emissive state. The emission maxima $(\lambda)$ of tCzphB-Ph and tCzphB-Fl in toluene were 524 $\mathrm{nm}$ and $532 \mathrm{~nm}$, respectively, which considerably red-shifted compared to $483 \mathrm{~nm}$ for DtBuCzB (Fig. 2b). The FWHMs of the emission spectra were both $21 \mathrm{~nm}$ for the two tCzphBs in toluene, which was narrower than $25 \mathrm{~nm}$ for $t$-DABNA and DtBuCzB. The emission spectra of the two tCzphBs blue-shifted and became narrower with a decrease in solvent polarity (Fig. 2c and Supplementary Fig. 1). In cyclohexane (cHex), the emissions of 
tCzphB-Ph and tCzphB-Fl had an FWHM value of $14 \mathrm{~nm}$ and CIE coordinates of $(0.15,0.77)$ and $(0.19,0.76)$, respectively, and these values were comparable to the best green-light emitting QDs. ${ }^{24-26}$ This was also the first time that the CIE y value of the PL spectrum of a molecule exceeded the NTSC green-light standard of $0.71 .{ }^{19-21,27-31}$ An analysis for the appearance of the narrow band in $\mathrm{tCzphB}-\mathrm{Ph}$ and $\mathrm{tCzphB-Fl}$ is given using the Huang-Rhys factor (Supplementary Note 1). Another analysis on the observation of wider PL band of tCzphB-Ph and tCzphB-Fl with FWHM of $21 \mathrm{~nm}$ in toluene compared with FWHM of $14 \mathrm{~nm}$ in cHex is given in Supplementary Note 2.

By doping into a classical host material 4,4'-Bis (N-carbazolyl)-1,1'-biphenyl (CBP) at a concentration of $2 \mathrm{wt} \%$, tCzphB-Ph and tCzphB-F1 exhibited their emission maxima at 529 $\mathrm{nm}$ and $537 \mathrm{~nm}$ with FWHM values of 30 and $33 \mathrm{~nm}$, respectively (Fig. 2c and Supplementary Fig. 1). The FWHM values of these two compounds in the carbazole-based host were considerably broader than in toluene, which was associated with the polarity of the carbazole units and the inhomogeneity of molecular aggregation. ${ }^{32}$ To inhibit the effect of solid-state solvation, a low polar sphere-like molecule, 2,2',7,7'-tetraphenyl-9,9'-spirobi[thioxanthene] (TPSS), was used to replace the commonly used carbazole derivatives as the host. The emission maxima of tCzphB-Ph and tCzphB-F1 in the TPSS host were comparable to those in CBP, and the FWHM values were reduced to 23 and $25 \mathrm{~nm}$, respectively (Fig. 2c). Because the highly rigid molecular frameworks suppressed structural deformation-induced nonradiative decay, the PL quantum yields (PLQYs, $\Phi)$ of tCzphB-Ph and tCzphB-Fl doped into the TPSS films (2 wt\%) reached 0.98 and 0.93 , respectively. In addition to the fluorescence decay with a lifetime $(\tau)$ of approximately $7 \mathrm{~ns}$, a 
delayed fluorescence component with a lifetime of hundreds of microseconds was observed (Fig. 2d, Table 1). Because the energy differences $\left(\Delta E_{\mathrm{ST}}\right)$ between the excited singlet $\left(\mathrm{S}_{1}\right)$ and triplet states $\left(T_{1}\right)$ estimated from the fluorescence and phosphorescence peaks (Fig. 2a) were as small as $0.08 \mathrm{eV}$ for $\mathrm{tCzphB}-\mathrm{Ph}$ and $0.07 \mathrm{eV}$ for $\mathrm{tCzphB}-\mathrm{Fl}$, the delayed fluorescence observed at RT was assigned to TADF. ${ }^{18}$

Single crystals of tCzphB-Ph and tCzphB-Fl were successfully obtained by slow methanol diffusion in dilute dichloride solutions. The crystal structures shown in Fig. 3a and Supplementary Fig. 2 revealed a planar skeleton in both molecules. The angles between the central phenyl ring and the surrounding phenyl rings were nearly 0 degrees. The phenyl and fluorenyl groups that connected to the spiro-carbons were almost perpendicular to the $\mathrm{BN}$ plane. Steric hindrance provided by these groups separated the BN planes of the adjacent molecules by a distance of $7.3 \AA$, which inhibited the $\pi-\pi$ interaction-induced fluorescence quenching and spectral broadening. The frontier molecular orbital distributions in tCzphB-Ph and tCzphB-Fl were simulated by density functional theory (DFT) at the B3LYP/6-31g (d, p) level. ${ }^{33}$ As shown in Fig. 3b and Supplementary Fig. 3, the HOMO and LUMO of tCzphBs were spatially separated, the same as those of $t$-DABNA and DtBuCzB. However, the delocalization range of the orbitals in tCzphBs was larger than the ranges of $t$-DABNA and $\mathrm{DtBuCzB}$. The vertical transition energy of $\mathrm{tCzphB}-\mathrm{Ph}$, which was calculated according to the time-dependent DFT (TD-DFT) method, was $2.61 \mathrm{eV}$. This value was lower than the values for $t$-DABNA and DtBuCzB, which were 3.07 and $2.84 \mathrm{eV}$, respectively (Supplementary Table 1). Because the electron-donating capacity of the fluorenyl group in tCzphB-Fl was higher than the isolated phenyl groups in tCzphB-Ph, the theoretical transition energy of 
tCzphB-Fl was $0.02 \mathrm{eV}$ lower than tCzphB-Ph, which was consistent with the experimental results (Table 1). The oscillator strengths $(f)$ of the $\mathrm{S}_{1}$ transitions in $\mathrm{tCzphB}-\mathrm{Ph}$ and tCzphB-F1 were both higher than 0.3 (Supplementary Table 1), ensuring high radiation rates and high PLQYs for these two emitters.

It is known to us that the electron-vibration coupling dominates the FWHM of the emission band when large-amplitude nuclear rearrangement in the $S_{1}$ state has been suppressed. ${ }^{10-13}$ To better understand the excellent color purity achieved by the tCzphBs, the vibrationally-resolved fluorescence spectra were simulated by TD-DFT at the PBE0/6-31g (d, p) level and compared with DtBuCzB (Fig. 3c, 3d, and Supplementary Fig. 4). ${ }^{34}$ The MR-TADF molecules suppressed spectral broadening due to the stretching vibrations of the $\pi$-bonds by alternately embedding electron-rich and electron-poor atoms between the benzene rings. However, because of repulsions by the hydrogen atoms in the adjacent phenyl rings, the tert-butylcarbazole groups and the central phenyl ring in $\mathrm{DtBuCzB}$ were not in the same plane. The calculated vibrationally-resolved fluorescence spectra showed that the twisting and stretching vibrations between the tert-butylcarbazole moieties and the central phenyl ring in the excited state considerably broadened the fluorescence spectrum of DtBuCzB. In the tCzphBs, the tert-butylcarbazole moiety and central phenyl ring were locked by a spiro-carbon atom, and consequently, vibrational relaxation in both the ground and excited states was significantly suppressed. The spectral simulations based on the Frank-Condon principle showed that the dominant vibration modes in the tCzphBs were the scissoring of phenyl or fluorenyl groups outside the main plane, and the rocking of the substituted tert-butyl groups, which were almost in the low frequency region (Supplementary Table 2) 
and had little effect on the fluorescence spectrum.

OLED performance. The thermal decomposition temperatures $\left(\mathrm{T}_{\mathrm{d}}\right)$ of $t \mathrm{CzphB}-\mathrm{Ph}$ and tCzphB-Fl were both over $400^{\circ} \mathrm{C}$, as recorded by thermogravimetric analysis (TGA) under a purged nitrogen atmosphere (Supplementary Fig. 5). The excellent thermal stability of these two compounds would allow for vacuum evaporation for device fabrication. The EL performance of the tCzphBs was then evaluated by the devices with a structure of ITO/HAT-CN (15 nm)/TAPC (70 nm)/TPSS:emitter (2 wt\%, $30 \mathrm{~nm}) /$ Bepp $_{2}(5 \mathrm{~nm}) / \mathrm{Bepp}_{2}:$ Liq (5 wt $\%, 30 \mathrm{~nm}) / \mathrm{Liq}(1 \mathrm{~nm}) / \mathrm{Al}$, where HAT-CN, TAPC, Bepp2, and Liq consisted of 1,4,5,8,9,11-hexaazatriphenylene-hexacarbonitrile, 1,1-bis [(di-4-tolylamino) phenyl] cyclohexane, bis [2-(2-hydroxyphenyl)-pyridine] beryllium, and 8-hydroxyquinolinolato-lithium, respectively (Supplementary Fig. 6-8). As shown in Fig. 4, both devices exhibited pure green emissions with an application voltage greater than $3.0 \mathrm{~V}$. The peaks, FWHMs, and CIE coordinate values of the EL emissions were $527 \mathrm{~nm}, 24 \mathrm{~nm}$, and $(0.21,0.75)$ for the tCzphB-Ph-based device, and $535 \mathrm{~nm}, 26 \mathrm{~nm}$, and $(0.26,0.72)$ for the tCzphB-Fl-based device, respectively. These values exceeded the requirements for the NTSC green-light standard $(\mathrm{CIE} \mathrm{y}=0.71)$ and set a new record for the highest CIE y values among all reported bottom-emitting OLEDs (Supplementary Table 3). ${ }^{19-21,}$ 27-31, 35, 36 Because tCzphB-Ph and tCzphB-Fl had sufficiently small $\Delta E_{\mathrm{ST}}$ values, enabling efficient up-conversion from $T_{1}$ to $S_{1}$, the external quantum efficiency (EQE) maximum values of their devices reached $29.3 \%$ and $26.2 \%$, respectively. However, the devices underwent serious efficiency roll-off as the current density increased, due to the long triplet state lifetimes of the two MR-TADF emitters (Table 1). Using the tCzphB-Ph-based device as an example, EQE 
dropped dramatically to $13.6 \%$ and $2.6 \%$ at luminescence values of 500 and $10,000 \mathrm{~cd} / \mathrm{cm}^{2}$, respectively (Fig. 4d).

To suppress the efficiency roll-off in the MR-TADF OLEDs, a phosphorescence sensitized TADF (PSTADF) OLED containing tCzphB-Ph as the emitter was fabricated. ${ }^{37}$ On the basis of the device structure mentioned above, the light-emitting layer was replaced with doped films consisting of BCz-o-TRZ:5 wt\% $\operatorname{Ir}\left(\right.$ ppy) 3 and BCz-o-TRZ:5 wt\% $\operatorname{Ir}(\mathrm{ppy})_{3}: 2 \mathrm{wt} \%$ tCzphB-Ph (Fig. 4), where BCz-o-TRZ (9-(2-(4,6-diphenyl-1,3,5-triazin-2-yl)phenyl)9'-phenyl-9H,9'H-3,3'-bicarbazole) and $\operatorname{Ir}($ ppy)3 (fac-tris (2-phenylpyridine) iridium) were the bipolar transport materials with a small $\Delta E_{\mathrm{ST}}$ and a classic green phosphor, respectively. ${ }^{38,39}$ The device without tCzphB-Ph had a unique EL spectrum of $\operatorname{Ir}(\mathrm{ppy})_{3}$ with an FWHM value of $58 \mathrm{~nm}$ and CIE coordinates of $(0.26,0.64)$. The device achieved an EQE maximum of $20.0 \%$ and an EQE of $18.0 \%$ at a luminance of $10,000 \mathrm{~cd} / \mathrm{m}^{2}$. The doping concentration of $5 \mathrm{wt} \%$ for $\operatorname{Ir}($ ppy) 3 achieved the optimized result (Supplementary Fig. 9). After doping $2 \mathrm{wt} \%$ tCzphB-Ph into the emitting layer of this traditional phosphorescence OLED (PHOLED), a sharp green emission from $\mathrm{tCzphB}-\mathrm{Ph}$ was detected instead of emission from $\operatorname{Ir}(\mathrm{ppy})_{3}$, indicating an efficient Förster resonance energy transfer (FRET) from $\operatorname{Ir}(\mathrm{ppy})_{3}$ to $\mathrm{tCzphB}-\mathrm{Ph}$ (Fig. 4a). ${ }^{40}$ In addition to the narrowed emission band with an FWHM of $27 \mathrm{~nm}$ and improved color purity with CIE coordinates of $(0.21,0.74)$, the double-doped device exhibited a greatly improved EQE with a maximum of $31.3 \%$, compared to the $\operatorname{Ir}(\mathrm{ppy})_{3}$-single-doped device. Specifically, the efficiency roll-off caused by phosphor-based triplet-triplet annihilation was significantly reduced in this double-doped device, due to the singlet harvest through a long-range FRET pathway (Fig. 4d). The PSTADF OLED maintained a high EQE value of 
$30.6 \%$ at a luminance of $10,000 \mathrm{~cd} / \mathrm{m}^{2}$ and achieved an ultrahigh luminescence of $4.6 \times 10^{5}$ $\mathrm{cd} / \mathrm{m}^{2}$, which was associated with a high EQE of $26.0 \%$. These were the best performance results for green-emitting OLEDs without the use of light out-coupling enhancement techniques (Supplementary Table 3). The increased EQE with respect to the control $\operatorname{Ir}(\text { ppy })_{3}$-based device was more closely related to the increased PLQY of the emitting layer. The PLQY values were 0.98 for the double-doped films containing $2 \mathrm{wt} \% \mathrm{tCzphB}-\mathrm{Ph}$, versus 0.67 for the $\operatorname{Ir}(\mathrm{ppy})_{3}$ doped BCz-o-TRZ film (5 wt\%).

\section{Discussion}

color-saturated green-emitting molecules were successfully obtained by locking all of the phenyl rings surrounding the $s p^{3}-\mathrm{N}$ atoms in MR-type blue-emitting $\mathrm{BN}$ molecules. The multi-lock strategy not only increased the planarity and conjugation of the emitter, but also suppressed intramolecular twisting and stretching vibrations, which consequently narrowed the emission band. In non-polar solvent cyclohexane, the FWHM and CIE y values of the emission spectra were $14 \mathrm{~nm}$ and 0.77 , respectively, and these values were comparable to state-of-the-art QDs, revealing great potential for EL and light conversion applications. The OLEDs containing these green emitters doped into a low polarity host material achieved a high EQE of $29.3 \%$, due to the high PLQY (0.98) and TADF properties of the emitters. The device exhibited a color-saturated green emission with an FWHM value of $24 \mathrm{~nm}$ and CIE coordinates of $(0.21,0.75)$, and was the purest green bottom-emitting OLED ever reported. By doping these innovative green emitters into PHOLEDs based on classical green phosphor $\operatorname{Ir}(\text { ppy })_{3}$, the EQE increased from $18.0 \%$ to $30.6 \%$ at a luminescence of $10,000 \mathrm{~cd} / \mathrm{m}^{2}$, and the spectral FWHM narrowed from 58 to $27 \mathrm{~nm}$. Because phosphor-based triplet-triplet 
annihilation was suppressed by the FRET pathway, the PSTADF OLED exhibited a reduced efficiency roll-off compared to the control PHOLED, and achieved an extremely high luminescence of $4.6 \times 10^{5} \mathrm{~cd} / \mathrm{m}^{2}$. Therefore, we believe that this multi-lock strategy for MR-TADF materials could be used for the development of narrowband organic emitters with full-color emissions, as well as high EL efficiency and stability.

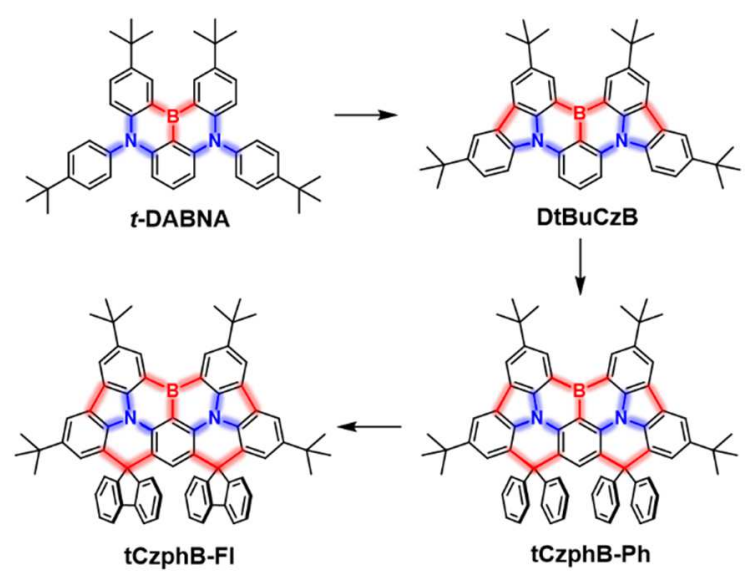

Fig. 1. Scheme showing the molecular design strategy and molecular structures of $t$-DABNA, DtBuCzB, tCzphB-Ph, and tCzphB-Fl. 

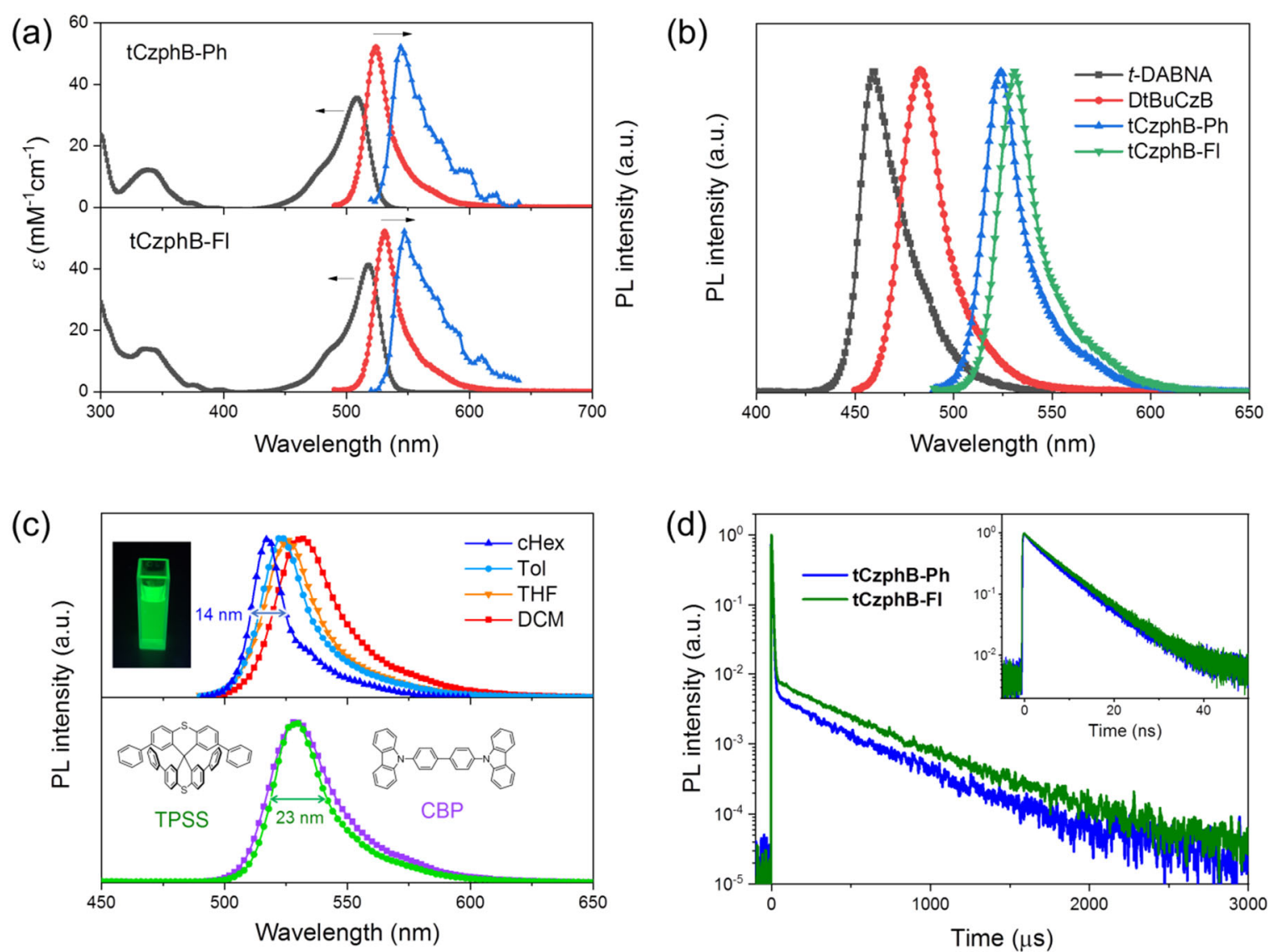

Fig. 2. (a) UV-vis absorption (gray line) and fluorescence (red line) spectra in toluene $\left(1 \times 10^{-5} \mathrm{M}\right)$ at RT and phosphorescence (100-1000 $\mu$ s component, blue line) spectra in toluene at $77 \mathrm{~K}$. (b) Fluorescence spectra of $t$-DABNA, DtBuCzB, tCzphB-Ph, and tCzphB-Fl in toluene at RT. (c) Fluorescence spectra of tCzphB-Ph in different solvents (cyclohexane, toluene, tetrahydrofuran, and dichloromethane) and organic semiconductor films at RT (inset: image of cyclohexane solution under UV light). (d) Transient PL decay spectra of tCzphB-Ph and tCzphB-Fl doped into the TPSS films (2 wt \%) at RT. 
(a)

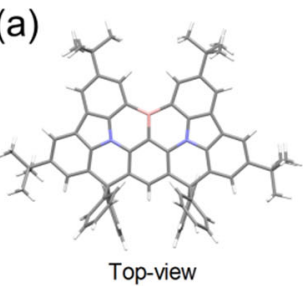

Top-view

(c)

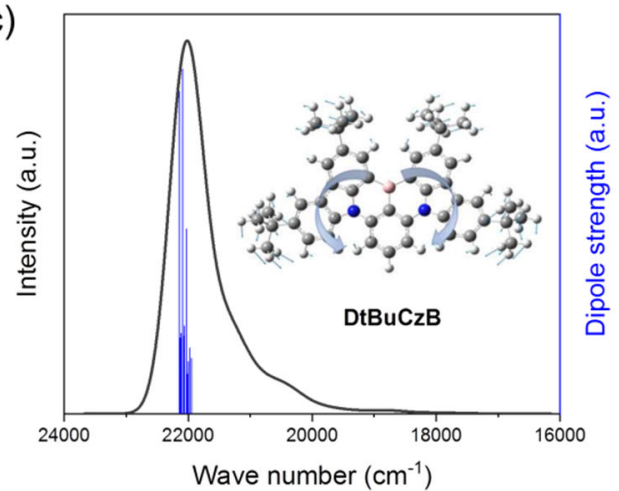

(b)

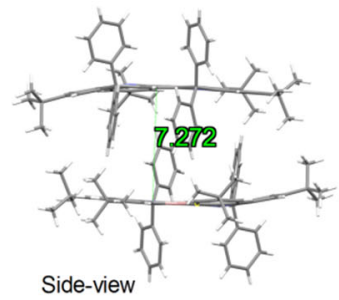

(d)

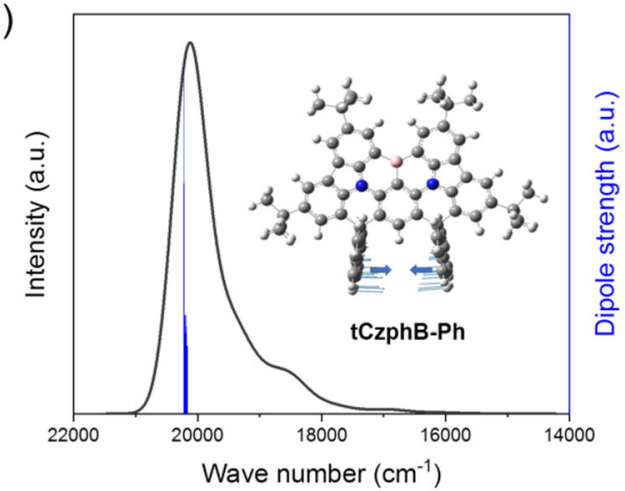

Fig. 3. (a, b) Crystal structure (a) and HOMO/LUMO distributions of (b) tCzphB-Ph. (c, d) Simulated vibrationally-resolved fluorescence spectra with dipole strengths of different vibrational modes for DtBuCzB (c) and tCzphB-Ph (d) Only several modes at high energies (Supplementary Table 2) are plotted by vertical lines. The simulated fluorescence spectra envelope is derived by mean of Gaussian function with half-width at half-maximum of $300 \mathrm{~cm}^{-1}$ for each vibration line which is calculated by the TD-DFT method, to compare the spectra observed in cHex. Inset: the molecular structures and domain vibration modes of $\mathrm{DtBuCzB}$ and $\mathrm{tCzphB}-\mathrm{Ph}$. 
(a)
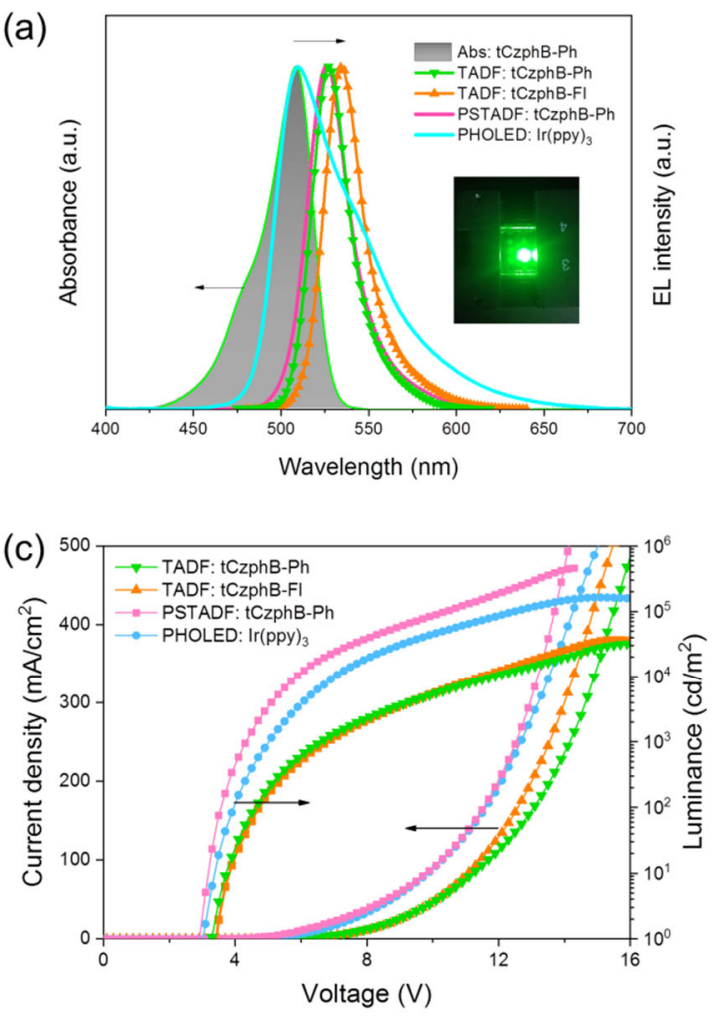
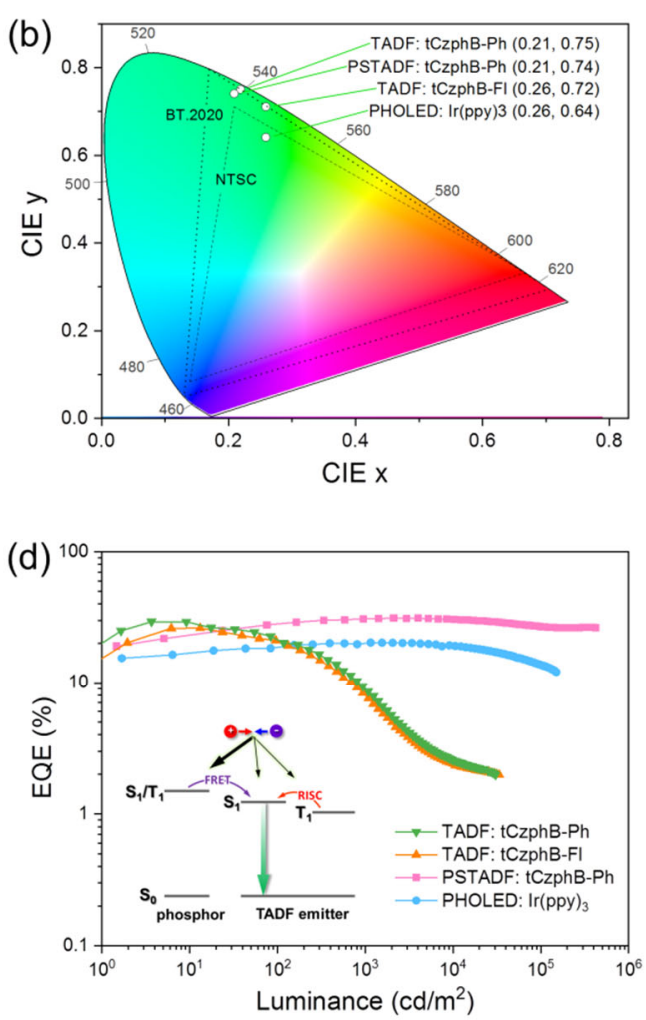

Fig. 4. (a) EL spectra of the TADF OLEDs containing tCzphB-Ph and tCzphB-Fl, PHOLED containing $\operatorname{Ir}(\mathrm{ppy})_{3}$, and the PSTADF OLED containing both $\operatorname{Ir}(\mathrm{ppy})_{3}$ and tCzphB-Ph (inset: image of the PSTADF OLED). (b) Corresponding 1931 CIE coordinates of the device emission. (c) Current density-voltage-luminance characteristics of the investigated OLEDs. (d) EQE-luminance characteristics of the investigated OLEDs. Inset: diagram of the energy transfer pathways in the PSTADF OLED.

Table 1: Photophysical parameters of tCzphB-Ph and tCzphB-Fl in the solutions and doped films.

\begin{tabular}{lcccccccccc}
\hline & $\lambda_{\mathrm{abs}}{ }^{a}$ & $\lambda_{\mathrm{PL}^{a}}$ & $\mathrm{FWHM}^{a}$ & $\Delta E_{\mathrm{ST}}{ }^{a}$ & $\mathrm{HOMO}_{2} / \mathrm{LMO}^{b}$ & $\lambda_{\mathrm{PL}}{ }^{c}$ & $\mathrm{FWHM}^{c}$ & $\tau_{\mathrm{p}}{ }^{c}$ & $\tau_{\mathrm{d}}{ }^{c}$ & $\Phi_{\mathrm{PL}^{c}}$ \\
& {$[\mathrm{~nm}]$} & {$[\mathrm{nm}]$} & {$[\mathrm{nm}]$} & {$[\mathrm{eV}]$} & {$[\mathrm{eV}]$} & {$[\mathrm{nm}]$} & {$[\mathrm{nm}]$} & {$[\mathrm{ns}]$} & {$[\mathrm{us}]$} & \\
\hline $\mathrm{tCzphB}-\mathrm{Ph}$ & 508 & 523 & 21 & 0.08 & $-5.15 /-2.72$ & 527 & 23 & 6.9 & 372 & 0.98 \\
$\mathrm{tCzphB-Fl}$ & 518 & 531 & 21 & 0.07 & $-5.12 /-2.73$ & 535 & 25 & 7.3 & 412 & 0.93 \\
\hline
\end{tabular}

${ }^{a}$ Measured in toluene with a concentration of $10^{-5} \mathrm{M} .{ }^{b}$ Determined from the cyclic voltammograms in dichloromethane and N,N-dimethylformamide for HOMO and LUMO energy levels, respectively (Supplementary Fig. 6). ${ }^{c}$ Measured in 2 wt\% doped TPSS films. 


\section{Methods}

General. All solvents and reactants used in this work were purchased from commercial resources and were used as received directly unless otherwise stated. All reactions were carried out under nitrogen in dry solvents. Compounds tCzphB-Ph and tCzphB-Fl were synthesized according to Supplementary Fig. 10 and purified by sublimation twice before device fabrication. Detailed synthesis procedures were showed in Supplementary Note 3. 2,2',7,7'-tetraphenyl-9,9'-spirobi [thioxanthene] (TPSS) was provided by Zhejiang Hongwu Technology Co.Ltd. The other organic materials for OLED fabrication were purchased from Jilin Optical and Electronic Materials Co. Ltd and were used as received.

Structure characterizations. Nuclear magnetic resonance spectroscopy (NMR) were recorded by a Bruker Avance III 400 spectrometer $\left({ }^{1} \mathrm{H}: 400 \mathrm{MHz},{ }^{13} \mathrm{C}: 101 \mathrm{MHz}\right)$ at $298 \mathrm{~K}$. The ${ }^{1} \mathrm{H}$ and ${ }^{13} \mathrm{C}$ NMR chemical shifts were expressed as $\delta$ downfield using tetramethylsilane (TMS) as internal reference. Mass spectra were recorded by Thermo Scientific ISQ ${ }^{\mathrm{TM}}$ EC single quadrupole mass spectrometer using positive ion electrospray ionization (ESI). Elemental analyses were performed using a EuroEA EA3000 and correlated with linear method.

Quantum Chemical Calculations. All calculations were performed using the Gaussian 16 program package. ${ }^{41}$ Ground state geometries were optimized by DFT calculations at the B3LYP/6-31G(d,p) level. By frequency analysis, structures were confirmed at the local minima of the potential surfaces. Vertical absorption energies and oscillator strengths $(f)$ of the $\mathrm{S}_{0} \rightarrow \mathrm{S}_{1}$ and $\mathrm{S}_{0} \rightarrow \mathrm{T}_{1}$ were calculated based on time-dependent DFT with the B3LYP function using $6-31 \mathrm{G}(\mathrm{d}, \mathrm{p})$ basis sets in gas phase. The models of molecular structure and the HOMO/LUMO iso-surface distribution were rendered by Multiwfn and VMD. ${ }^{33,42}$ For Franck-Condon analysis to calculate vibrationally-resolved PL spectra, the geometries of all molecules in their ground state $\left(\mathrm{S}_{0}\right)$ and excited state $\left(\mathrm{S}_{1}\right)$ were obtained by TD-DFT/PBE0/6-31G $(\mathrm{d}, \mathrm{p})$ in toluene solvent. 
Thermal properties. Thermogravimetric analysis (TGA) were performed by TA-Q50. The TGA curve was measured at heating rate of $10{ }^{\circ} \mathrm{C} / \mathrm{min}$ from RT to $500^{\circ} \mathrm{C}$ under nitrogen flow, after eliminating residual thermal history of compound.

Cyclic voltammetry measurements. Electrochemical properties were measured by a CHI-600E electrochemical analyzer S-20 in a gas-tight three-electrode cell at RT. A glassy carbon working electrode $(\Phi=5.0 \mathrm{~mm})$, a platinum wire auxiliary electrode, and an $\mathrm{Ag} / \mathrm{Ag}^{+}$ reference electrode with $0.1 \mathrm{M} \mathrm{AgNO}_{3}$ and $0.1 \mathrm{M}$ tetrabutylammonium hexafluorophosphate (TBAP) in acetonitrile were used. The oxidation processes were measured by scanning the potential at a scan rate of $100 \mathrm{mV} / \mathrm{s}$ in dichloromethane (DCM) with $0.1 \mathrm{M}$ TBAP as supporting electrolyte. The reduction processes were measured by scanning the potential at a scan rate of $100 \mathrm{mV} / \mathrm{s}$ in N,N-dimethylformamide (DMF) with $0.1 \mathrm{M}$ TBAP as supporting electrolyte. Before the measurements, solutions were degassed by purging with $\mathrm{N}_{2}$ for approximately 1 minute (min). The ferrocene couple $\mathrm{Fc} / \mathrm{Fc}^{+}$was served as the external reference.

Photophysical measurements. UV-VIS absorption spectra were measured by a Shimadzu UV2600 UV/VIS spectrophotometer. Photoluminescence (PL) spectra and quantum yield of the samples were measured using a QM-40 spectrofluorometer (PTI, Horiba) equipped with a $150 \mathrm{~W}$ xenon lamp and an integrating sphere. Time-resolved fluorescence (1 ns) and phosphorescence (100-1000 $\mu \mathrm{s})$ spectra and microsecond transient decay spectra were recorded by a PTI TimeMaster fluorimeter equipped with a PTI nitrogen laser (GL-3300, $\lambda=$ $337 \mathrm{~nm}$, pulse width $\approx 1 \mathrm{~ns}$, pulse energy $=1.45 \mathrm{~mJ}$ ). Nanosecond transient decay spectra were recorded on Horiba DeltaFlex modular lifetime measurement system patented the time-correlated single-photon counting (TCSPC) technique and equipped with a diode laser as the excitation source $(\lambda=370 \mathrm{~nm}$, pulse width $\approx 50 \mathrm{ps}$, repetition rate $=20.00 \mathrm{kHz})$.

X-ray Crystallography. X-ray diffraction data were measured at $170 \mathrm{~K}$ on a Bruker APEX-II CCD diffractometer using graphite-monochromated Mo-K $\alpha$ radiation $(\lambda=0.71073 \AA)$ from a rotating anode generator. Using the Olex2 program, ${ }^{43}$ the structures were solved with the 
ShelXT structure solution program using Intrinsic Phasing and refined with the ShelXL refinement package using Least Squares minimisation. ${ }^{44}$ All Hydrogen atoms on carbons were placed in calculated positions and refined using the riding model. All non-hydrogen atoms were refined with anisotropic displacement parameters. Solvent molecules in crystal were squeezed. The crystal data as well as the details of data collection and refinement are summarized in Supplementary Table 4 and 5.

OLED fabrication and measurements. The organic layers and metal layer were thermally evaporated on the cleaned indium-tin-oxide (ITO) glass substrate under vacuum $\left(<4 \times 10^{-4} \mathrm{~Pa}\right)$ with $1 \AA /$ s deposition rate for organic layers and $4 \AA /$ s for aluminum cathode. The active area of the devices is $9 \mathrm{~mm}^{2}(3 \mathrm{~mm} \times 3 \mathrm{~mm})$. The current density-voltage- luminance characteristics of OLEDs were measured with a Keithey 2400 Source meter and a Keithey 2000 Source multimeter equipped with a calibrated silicon photodiode. The electroluminescence (EL) spectra were recorded using a multichannel spectrometer (PMA12, Hamamatsu Photonics). The external quantum efficiency can be calculated from the combination of luminance, current density and EL spectrum when Lambertian emission was assumed.

\section{Data Availability}

The authors declare that the main data supporting the findings of this study are available within the article and its Supplementary Information files. Extra data are available from the corresponding author upon request.

\section{Crystallographic data}

The new crystallographic structures of target molecules are available within the accompanied files. 


\section{References}

1. Colvin, V. L., Schlamp, M. C. \& Alivisatos, A. P. Light-emitting diodes made from cadmium selenide nanocrystals and a semiconducting polymer. Nature 370, 354-357 (1994).

2. Coe, S., Woo, W.-K., Bawendi, M. \& Bulović, V. Electroluminescence from single monolayers of nanocrystals in molecular organic devices. Nature 420, 800-803 (2002).

3. Dai, X., Deng, Y., Peng, X. \& Jin, Y. Quantum-dot light-emitting diodes for large-area displays: towards the dawn of commercialization. Adv. Mater. 29, 1607022 (2017).

4. Yang, Z. et al. Recent advances in quantum dot-based light-emitting devices: Challenges and possible solutions. Mater. Today 24, 69-93 (2019).

5. Yokoyama, H. Physics and device applications of optical microcavities. Science 256, 66-70 (1992).

6. Köhler, A., Bässler, H. Electronic Processes in Organic Semiconductors: An Introduction. (Wiley-VCH, Weinheim, 2015).

7. Grabowski, Z. R., Rotkiewicz, K. \& Rettig, W. Structural changes accompanying intramolecular electron transfer: Focus on twisted intramolecular charge-transfer states and structures. Chem. Rev. 103, 3899-4032 (2003).

8. Kaji, H. et al. Purely organic electroluminescent material realizing $100 \%$ conversion from electricity to light. Nat. Commun. 6, 8476 (2015).

9. Rajamalli, P. et al. New molecular design concurrently providing superior pure blue, thermally activated delayed fluorescence and optical out-coupling efficiencies. J. Am. Chem. Soc. 139, 1094810951 (2017).

10. Gierschner, J., Mack, H.-G., Lüer, L. \& Oelkrug, D. Fluorescence and absorption spectra of oligophenylenevinylenes: Vibronic coupling, band shapes, and solvatochromism. J. Chem. Phys. 116, 8596-8609 (2002). 
11. Santoro, F., Lami, A., Improta, R., Bloino, J. \& Barone, V. Effective method for the computation of optical spectra of large molecules at finite temperature including the Duschinsky and Herzberg-Teller effect: The Qx band of porphyrin as a case study. J. Chem. Phys. 128, 224311 (2008).

12. Ha, J. M. et al. Rational molecular design of azaacene-based narrowband green-emitting fluorophores: Modulation of spectral bandwidth and vibronic transitions. ACS Appl. Mater. Interfaces 13, 26227$26236(2021)$.

13. Kong, F.-F. et al. Probing intramolecular vibronic coupling through vibronic-state imaging. Nat. Commun. 12, 1280 (2021).

14. Xu, Y. et al. Molecular engineered conjugated polymer with high thermal conductivity. Sci. Adv. 4, eaar3031 (2018).

15. Hatakeyama, T. et al. Ultrapure blue thermally activated delayed fluorescence molecules: Efficient HOMO-LUMO separation by the multiple resonance effect. Adv. Mater. 28, 2777-2781 (2016).

16. Kondo, Y. et al. Narrowband deep-blue organic light-emitting diode featuring an organoboron-based emitter. Nat. Photonics 13, 678-682 (2019).

17. Suresh, S. M., Hall, D., Beljonne, D., Olivier, Y. \& Zysman-Colman, E. Multiresonant thermally activated delayed fluorescence emitters based on heteroatom-doped nanographenes: Recent advances and prospects for organic light-emitting diodes. Adv. Funct. Mater. 30, 1908677 (2020).

18. Pershin, A. et al. Highly emissive excitons with reduced exchange energy in thermally activated delayed fluorescent molecules, Nat. Commun. 10, 597 (2019).

19. Zhang, Y. et al. Multi-resonance induced thermally activated delayed fluorophores for narrowband green OLEDs. Angew. Chem. Int. Ed. 58, 16912-16917 (2019).

20. Xu, Y. et al. Molecular-structure and device-configuration optimizations toward highly efficient green electroluminescence with narrowband emission and high color purity. Adv. Opt. Mater. 8, 1902142 
(2020).

21. Zhang, Y. et al. Achieving pure green electroluminescence with CIEy of 0.69 and EQE of $28.2 \%$ from an aza-fused multi-resonance emitter. Angew. Chem. Int. Ed. 59, 17499-17503 (2020).

22. Wang, Y. et al. A periphery cladding strategy to improve the performance of narrowband emitters, achieving deep-blue OLEDs with CIEy $<0.08$ and external quantum efficiency approaching $20 \%$. Org. Electron. 97, 106275 (2021).

23. Han, S. H., Jeong, J. H., Yoo, J. W. \& Lee, J. Y. Ideal blue thermally activated delayed fluorescence emission assisted by a thermally activated delayed fluorescence assistant dopant through a fast reverse intersystem crossing mediated cascade energy transfer process. J. Mater. Chem. C 7, 3082-3089 (2019).

24. Bae, W. K. et al. Highly efficient green-light-emitting diodes based on CdSe@ZnS quantum dots with a chemical-composition gradient. Adv. Mater. 21, 1690-1694 (2009).

25. Kwak, J. et al. Bright and efficient full-color colloidal quantum dot light-emitting diodes using an inverted device structure. Nano Lett. 12, 2362-2366 (2012).

26. Lee, K.-H. et al. Over $40 \mathrm{~cd} / \mathrm{A}$ efficient green quantum dot electroluminescent device comprising uniquely large-sized quantum dots. ACS Nano 8, 4893-4901 (2014).

27. Lee, M.-T. et al. Efficient green coumarin dopants for organic light-emitting devices. Org. Lett. 6, $1241-1244(2004)$.

28. Fukagawa, H., Oono, T., Iwasaki, Y., Hatakeyama, T. \& Shimizu, T. High-efficiency ultrapure green organic light-emitting diodes. Mater. Chem. Front. 2, 704-709 (2018).

29. Song, X., Zhang, D., Zhang, Y., Lu, Y. \& Duan, L. Strategically modulating carriers and excitons for efficient and stable ultrapure-green fluorescent OLEDs with a sterically hindered BODIPY dopant. Adv. Opt. Mater. 8, 2000483 (2020). 
30. Xu, Y. et al. Constructing charge-transfer excited states based on frontier molecular orbital engineering: Narrowband green electroluminescence with high color purity and efficiency. Angew. Chem. Int. Ed. 59, 17442-17446 (2020).

31. Yang, M., Park, I. S. \& Yasuda, T. Full-color, narrowband, and high-efficiency electroluminescence from boron and carbazole embedded polycyclic heteroaromatics. J. Am. Chem. Soc. 142, 19468-19472 (2020).

32. Deng, C., Zhang, L., Wang, D., Tsuboi, T. \& Zhang, Q. Exciton- and polaron-induced reversible dipole reorientation in amorphous organic semiconductor films. Adv. Opt. Mater. 7, 1801644 (2019).

33. Lu, T. \& Chen, F. Multiwfn: A multifunctional wavefunction analyzer. J. Comput. Chem. 33, 580-592 (2012).

34. Fortino, M. et al. On the simulation of vibrationally resolved electronic spectra of medium-size molecules: the case of styryl substituted BODIPYs. Phys. Chem. Chem. Phys. 21, 3512-3526 (2019).

35. Uoyama, H., Goushi, K., Shizu, K., Nomura, H. \& Adachi, C. Highly efficient organic light-emitting diodes from delayed fluorescence. Nature 492, 234 (2012).

36. Wu, T.-L. et al. Diboron compound-based organic light-emitting diodes with high efficiency and reduced efficiency roll-off. Nat. Photon. 12, 235-240 (2018).

37. Lee, K. H. \& Lee, J. Y. Phosphor sensitized thermally activated delayed fluorescence organic light-emitting diodes with ideal deep blue device performances. J. Mater. Chem. C 7, 8562-8568 (2019).

38. Zheng, X. et al. Expanding the hole delocalization range in excited molecules for stable organic light-emitting diodes employing thermally activated delayed fluorescence. J. Mater. Chem. C 8, $10021-10030$ (2020).

39. Baldo, M. A., Lamansky, S., Burrows, P. E., Thompson, M. E. \& Forrest, S. R. Very high-efficiency 
green organic light-emitting devices based on electrophosphorescence. Appl. Phys. Lett. 75, 4-6 (1999).

40. Baldo, M. A., Thompson, M. E. \& Forrest, S. R. High-efficiency fluorescent organic light-emitting devices using a phosphorescent sensitizer. Nature 403, 750-753 (2000).

41. Frisch, M. J. et al. Gaussian 16 Revision A.03. (Gaussian Inc. Wallingford CT, 2016).

42. Humphrey, W., Dalke, A. \& Schulten, K. VMD: Visual molecular dynamics. J. Mol. Graphics 14, 3338 (1996).

43. Dolomanov, O. V., Bourhis, L. J., Gildea, R. J., Howard, J. a. K. \& Puschmann, H. OLEX2: a complete structure solution, refinement and analysis program. J. Appl. Cryst. 42, 339-341 (2009).

44. Sheldrick, G. M. Crystal structure refinement with SHELXL. Acta. Cryst. C 71, 3-8 (2015).

\section{Acknowledgements}

This work was supported by the National Natural Science Foundation of China (Grant No. $51873183)$

\section{Ethics declarations}

Competing interests

The authors declare no competing financial interests.

\section{Tables of context.}


Combination of multiple resonance effect and multi-lock strategy enables green-emitting thermally activated delayed fluorescent molecules to exhibit super-high quantum efficiency $(0.98)$ and color purity $(\mathrm{CIE} y=0.77)$ that are comparable to the best quantum dots currently available. Doping these emitters into green-emitting phosphorescence organic light-emitting diodes endowed the device with a Broadcast Service Television 2020 color-gamut, 50\% improved external quantum efficiency, and an extremely high luminescence of $4.6 \times 10^{5} \mathrm{~cd} / \mathrm{m}^{2}$.

ToC figure

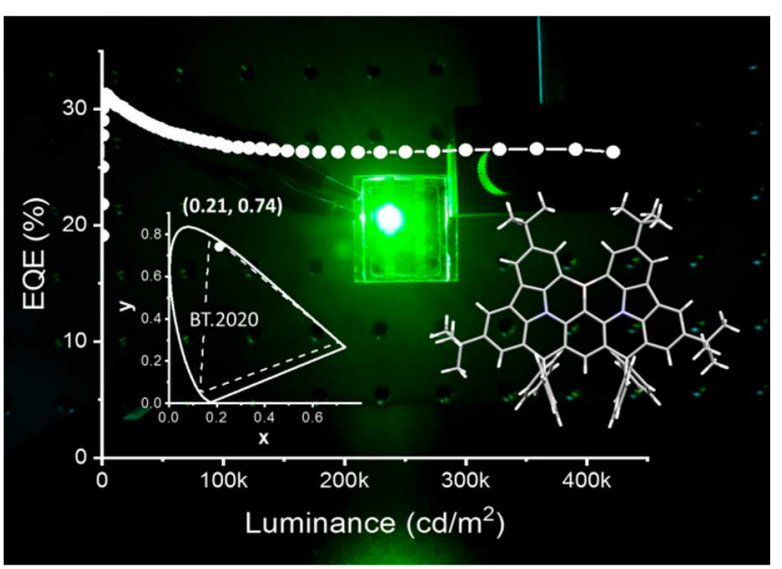




\section{Supplementary Files}

This is a list of supplementary files associated with this preprint. Click to download.

- Supportinglnformation.pdf 